\title{
Construction of second order slope rotatable designs under tri-diagonal correlated structure of errors using central composite designs
}

\author{
K. Rajyalakshmi *, B. Re. Victorbabu \\ Department of Statistics, Acharya Nagarjuna University, Guntur-522510, India \\ *Corresponding author E-mail: rajyalakshmi_kottapalli@yahoo.com
}

Copyright () 2014 Rajyalakshmi and Victorbabu. This is an open access article distributed under the Creative Commons Attribution License, which permits unrestricted use, distribution, and reproduction in any medium, provided the original work is properly cited.

\begin{abstract}
In this paper, second order slope rotatable design (SOSRD) under tri-diagonal correlated structure of errors using central composite designs (CCD) is suggested.

Keywords: Response Surface Designs, Rotatable Designs, Slope Rotatable Designs, Second Order Slope Rotatable Designs (SOSRD), Tri-Diagonal Correlated Errors.
\end{abstract}

\section{Introduction}

Box and Hunter [1] introduced rotatable designs for the exploration of response surface designs. Panda and Das [9] introduced first order rotatable designs with correlated errors. Das [2] introduced robust second order rotatable designs (RSORD). Das [3], [4] studied RSORD.

In response surface methodology, good estimation of the derivatives of the response function may be as important or perhaps more important than estimation of mean response. Estimation of differences in responses at two different points in the factor space will often be of great importance. If difference in responses at two points close together is of interest then estimation of local slope (rate of change) of the response is required. Estimation of slopes occurs frequently in practical situations. For instance, there are cases in which we want to estimate rate of reaction in chemical experiment, rate of change in the yield of a crop to various fertilizer doses, rate of disintegration of radioactive material in animal etc, (Park [10]).

Hader and Park [8] introduced slope rotatable central composite designs (SRCCD). Park [10] studied a class of multifactor designs for estimating the slope of response surfaces. Das [5] introduced slope rotatability with correlated errors. Das [6] studied measure of robust slope rotatability for second order response surface experimental designs. Das et al [7] suggested on D -optimal robust second order slope rotatable designs (RSOSRD). In this paper following the works of Das [4], [5], SOSRD under tri-diagonal correlated structure of errors using CCD is suggested. Further, the variance of the estimated slopes for different values of the tri-diagonal correlated coefficient $\rho$ ( -0.9 to 0.9$)$ for "v factors 2 to 8 " using CCD is studied.

\section{Conditions for SORD under tri-diagonal correlated structure of errors (cf. Das [4], Das et al [7])}

A second order response surface design $\mathrm{D}=\left(\left(\mathrm{x}_{\mathrm{iu}}\right)\right)$ for fitting

$Y_{u}=b_{0}+\sum_{i=1}^{v} b_{i} x_{i u}+\sum_{i=1}^{v} b_{i i} x_{i u}^{2}+\sum_{i=1}^{v} \sum_{i \leq j=1}^{v} b_{i j} x_{i u} x_{j u}+e_{u}$

where $x_{i u}$ denotes the level of the $i^{\text {th }}$ factor $(i=1,2, \ldots, v)$ in the $u^{\text {th }}$ run $(u=1,2, \ldots, 2 n)$ of the experiment, $e_{u}$ 's are correlated random errors, is said to be a SORD under tri-diagonal correlated structure of errors, if the variance of the 
estimated response of $\widehat{Y}_{u}$ from the fitted surface is only a function of the distance, $\left(d^{2}=\sum x_{i}^{2}\right)$ of the point $\left(x_{1 u}, x_{2 u}, \ldots, x_{v u}\right)$ from the origin (centre) of the design. Such a spherical variance function for estimation of responses in the second order response surface is achieved if the design points satisfy the following conditions.

$\sum_{\mathrm{u}=1}^{2 \mathrm{n}} \prod_{\mathrm{i}=1}^{\mathrm{v}} \mathrm{x}_{\mathrm{iu}}^{\alpha_{\mathrm{i}}}=0$ if any $\alpha_{\mathrm{i}}$ is odd, for $\sum \alpha_{\mathrm{i}} \leq 4$

$\sum_{\mathrm{u}=1}^{2 \mathrm{n}} \mathrm{x}_{\mathrm{iu}}^{2}=$ constant $=2 \mathrm{n} \lambda_{2}$

$\sum_{\mathrm{u}=1}^{2 \mathrm{n}} \mathrm{x}_{\mathrm{iu}}^{4}=$ constant $=3(2 \mathrm{n}) \lambda_{4}$, for all $\mathrm{i}$

$\sum_{\mathrm{u}=1}^{2 \mathrm{n}} \mathrm{x}_{\mathrm{iu}}^{2} \mathrm{x}_{\mathrm{ju}}^{2}=$ constant $=2 \mathrm{n} \lambda_{4}$, for all $\mathrm{i} \neq \mathrm{j}$

$\sum_{\mathrm{u}=1}^{2 \mathrm{n}} \mathrm{x}_{\mathrm{iu}}^{4}=3 \sum_{\mathrm{u}=1}^{2 \mathrm{n}} \mathrm{x}_{\mathrm{iu}}^{2} \mathrm{x}_{\mathrm{ju}}^{2}$

$\frac{\lambda_{4}}{\lambda_{2}^{2}}>\frac{\mathrm{v}(1-\rho)}{(\mathrm{v}+2)} \quad$ (non - singularity condition)

Where $\lambda_{2}$ and $\lambda_{4}$ constants and the summation are is over the design points.

The variances and covariances of the estimated parameters are,

$\mathrm{V}\left(\hat{\mathrm{b}}_{0}\right)=\frac{\lambda_{4}(\mathrm{v}+2)(1+\rho) \sigma^{2}}{2 \mathrm{n} \Delta}$

$\mathrm{V}\left(\hat{\mathrm{b}}_{\mathrm{i}}\right)=\frac{\sigma^{2}\left(1-\rho^{2}\right)}{2 \mathrm{n} \lambda_{2}}$

$\mathrm{V}\left(\hat{\mathrm{b}}_{\mathrm{ij}}\right)=\frac{\sigma^{2}\left(1-\rho^{2}\right)}{2 \mathrm{n} \lambda_{4}}$

$\mathrm{V}\left(\hat{\mathrm{b}}_{\mathrm{ii}}\right)=\frac{\sigma^{2}\left(1-\rho^{2}\right)\left[\lambda_{4}(\mathrm{v}+1)-(\mathrm{v}-1) \lambda_{2}^{2}(1-\rho)\right]}{2(2 \mathrm{n}) \lambda_{4} \Delta}$

$\operatorname{Cov}\left(\hat{\mathrm{b}}_{0}, \hat{\mathrm{b}}_{\mathrm{ii}}\right)=\frac{-\lambda_{2} \sigma^{2}\left(1-\rho^{2}\right)}{2 \mathrm{n} \Delta}$

$\operatorname{Cov}\left(\hat{\mathrm{b}}_{\mathrm{ii}}, \hat{\mathrm{b}}_{\mathrm{jj}}\right)=\frac{\sigma^{2}\left(1-\rho^{2}\right)\left[\lambda_{2}^{2}(1-\rho)-\lambda_{4}\right]}{2(2 \mathrm{n}) \lambda_{4} \Delta}$

Where $\Delta=\left[\lambda_{4}(v+2)-v \lambda_{2}^{2}(1-\rho)\right]$ and other covariances are zero.

\section{Conditions for SOSRD under tri-diagonal correlated structure of errors}

A second order response surface design $\mathrm{D}=\left(\left(\mathrm{x}_{\mathrm{iu}}\right)\right)$ for fitting $\mathrm{Y}_{\mathrm{u}}=\mathrm{b}_{0}+\sum_{\mathrm{i}=1}^{\mathrm{v}} \mathrm{b}_{\mathrm{i}} \mathrm{x}_{\mathrm{iu}}+\sum_{\mathrm{i}=1}^{\mathrm{v}} \mathrm{b}_{\mathrm{ii}} \mathrm{x}_{\mathrm{iu}}^{2}+\sum_{\mathrm{i}=1}^{\mathrm{v}} \sum_{\mathrm{i} \leq \mathrm{j}=1}^{\mathrm{v}} \mathrm{b}_{\mathrm{ij}} \mathrm{x}_{\mathrm{iu}} \mathrm{x}_{\mathrm{ju}}+\mathrm{e}_{\mathrm{u}}$ where $\mathrm{x}_{\mathrm{iu}}$ denotes the level of the $\mathrm{i}^{\text {th }}$ factor $(\mathrm{i}=1,2, \ldots, \mathrm{v})$ in the $\mathrm{u}^{\text {th }}$ run $(\mathrm{u}=1,2, \ldots, 2 \mathrm{n})$ of the experiment, $\mathrm{e}_{\mathrm{u}}$ 's are correlated random errors, is said to be a SOSRD under tri-diagonal correlated structure of errors, if the variance of the estimate of first order partial derivative of $Y_{u}\left(x_{1}, x_{2}, \ldots, x_{v}\right)$ with respect to each of independent variable $\left(x_{i}\right)$ is only a function of the distance $\left(\mathrm{d}^{2}=\sum_{\mathrm{i}=1}^{\mathrm{v}} \mathrm{x}_{\mathrm{i}}^{2}\right)$ of the point $\left(\mathrm{x}_{1}, \mathrm{x}_{2}, \ldots, \mathrm{x}_{\mathrm{v}}\right)$ from the origin (centre of the design). Such a spherical variance function for estimation of slopes in the second order response surface is achieved if the design points satisfy the following conditions (cf. Das [4, 5]).

The necessary and sufficient conditions for second order rotatability under the tri-diagonal correlated variance covariance structure, for all values of $\rho$, in the model (9) are

(i) $\sum_{u=1}^{2 n} x_{j u}=0$,
(ii) $\sum_{u=1}^{2 n} x_{j u} x_{l u}=0,1 \leq j \leq v ;$
(iii) $\sum_{u=1}^{2 n} x_{i u} x_{j u}-\rho\left\{\sum_{u=1}^{n} x_{i(n+u)} x_{j u}+\sum_{u=1}^{n} x_{i u} x_{j(n+u)}\right\}=0, \quad 1 \leq i \neq j \leq v ;$
(iv) $\sum_{u=1}^{2 n} x_{i u}^{2} x_{j u}-\rho\left\{\sum_{u=1}^{n} x_{i(n+u)}^{2} x_{j u}+\sum_{u=1}^{n} x_{i u}^{2} x_{j(n+u)}\right\}=0, \quad 1 \leq i, j \leq v ;$
(v) $\sum_{u=1}^{2 n} x_{i u} x_{j u} x_{l u}-\rho\left\{\sum_{u=1}^{n} x_{i(n+u)} x_{j(n+u)} x_{l u}+\sum_{u=1}^{n} x_{i u} x_{j u} x_{l(n+u)}\right\}=0$,
(vi) $\sum_{u=1}^{2 n} x_{i u}^{2} x_{j u} x_{l u}-\rho\left\{\sum_{u=1}^{n} x_{i(n+u)}^{2} x_{j u} x_{l u}+\sum_{u=1}^{n} x_{i u}^{2} x_{j(n+u)} x_{l(n+u)}\right\}=0$,

(vii) $\sum_{\mathrm{u}=1}^{2 \mathrm{n}} \mathrm{x}_{\mathrm{iu}} \mathrm{x}_{\mathrm{ju}} \mathrm{x}_{\mathrm{lu}} \mathrm{x}_{\mathrm{tu}}-\rho\left\{\sum_{\substack{\mathrm{u}=1 \\ 1 \leq \mathrm{i}, \mathrm{l}<j, t \leq v ;(\mathrm{i}, \mathrm{j}) \neq(\mathrm{l}, \mathrm{t})}}^{\mathrm{n}} \mathrm{x}_{\mathrm{i}(\mathrm{n}+\mathrm{u})} \mathrm{x}_{\mathrm{j}(\mathrm{n}+\mathrm{u})} \mathrm{x}_{\mathrm{lu}} \mathrm{x}_{\mathrm{tu}}+\sum_{\mathrm{u}=1}^{\mathrm{n}} \mathrm{x}_{\mathrm{iu}} \mathrm{x}_{\mathrm{ju}} \mathrm{x}_{\mathrm{l}(\mathrm{n}+\mathrm{u})} \mathrm{x}_{\mathrm{t}(\mathrm{n}+\mathrm{u})}\right\}=0$,

$1 \leq \mathrm{i}, \mathrm{j}<l \leq v ;(\mathrm{j}, \mathrm{l}) \neq(\mathrm{i}, \mathrm{i})$

$\sum_{\mathrm{u}=1}^{2 \mathrm{n}} \mathrm{x}_{\mathrm{iu}}^{2}=$ constant $=2 \mathrm{n} \lambda_{2}$ 
$\sum_{\mathrm{u}=1}^{2 \mathrm{n}} \mathrm{x}_{\mathrm{iu}}^{4}=$ constant $=\mathrm{c}(2 \mathrm{n}) \lambda_{4}$, for all $\mathrm{i}$

$\sum_{\mathrm{u}=1}^{2 \mathrm{n}} \mathrm{x}_{\mathrm{iu}}^{2} \mathrm{x}_{\mathrm{ju}}^{2}=$ constant $=2 \mathrm{n} \lambda_{4}$, for all $\mathrm{i} \neq \mathrm{j}$

$\sum_{\mathrm{u}=1}^{2 \mathrm{n}} \mathrm{x}_{\mathrm{iu}}^{4}=\mathrm{c} \sum_{\mathrm{u}=1}^{2 \mathrm{n}} \mathrm{x}_{\mathrm{iu}}^{2} \mathrm{x}_{\mathrm{ju}}^{2}$

Using (11) (12) and (13) the design parameters of the tri-diagonal correlated structure are as follows:

$(1-\rho)\left\{\sigma^{2}\left(1-\rho^{2}\right)\right\}^{-1} \sum_{u=1}^{2 n} x_{j u}^{2}=\frac{2 n \lambda_{2}(1-\rho)}{\sigma^{2}\left(1-\rho^{2}\right)}$

$\left\{\sigma^{2}\left(1-\rho^{2}\right)\right\}^{-1}\left[\sum_{u=1}^{2 n} x_{i u}^{2}-2 \rho \sum_{u=1}^{n} x_{i u} x_{i(n+u)}\right]=\frac{2 n \lambda_{2}}{\sigma^{2}\left(1-\rho^{2}\right)}, 1 \leq \mathrm{i} \leq v$

$\left\{\sigma^{2}\left(1-\rho^{2}\right)\right\}^{-1}\left[\sum_{u=1}^{2 n} x_{i u}^{4}-2 \rho \sum_{u=1}^{n} x_{i u}^{2} x_{i(n+u)}^{2}\right]=c\left(\frac{2 n \lambda_{4}}{\sigma^{2}\left(1-\rho^{2}\right)}\right) ; 1 \leq \mathrm{i} \leq v$

$\left\{\sigma^{2}\left(1-\rho^{2}\right)\right\}^{-1}\left[\sum_{u=1}^{2 n} x_{i u}^{2} x_{j u}^{2}-\rho\left[\sum_{u=1}^{n} x_{i(n+u)}^{2} x_{j u}^{2}+\sum_{u=1}^{n} x_{i u}^{2} x_{j(n+u)}^{2}\right]\right]=\frac{2 n \lambda_{4}}{\sigma^{2}\left(1-\rho^{2}\right)} ; 1 \leq i \neq j \leq v$

$\left\{\sigma^{2}\left(1-\rho^{2}\right)\right\}^{-1}\left[\sum_{u=1}^{2 n} x_{i u}^{2} x_{j u}^{2}-2 \rho \sum_{u=1}^{n} x_{i u} x_{j u} x_{i(n+u)} x_{j(n+u)}\right]=\frac{2 n \lambda_{4}}{\sigma^{2}\left(1-\rho^{2}\right)} ; 1 \leq i<j \leq v$

From (17), (18) and (19),

$$
\begin{aligned}
\left\{\sigma^{2}\left(1-\rho^{2}\right)\right\}^{-1}\left[\sum_{u=1}^{2 n} x_{i u}^{4}-2 \rho \sum_{u=1}^{n} x_{i u}^{2} x_{i(n+u)}^{2}\right] \\
=2\left(\left\{\sigma^{2}\left(1-\rho^{2}\right)\right\}^{-1}\left[\sum_{u=1}^{2 n} x_{i u}^{2} x_{j u}^{2}-2 \rho \sum_{u=1}^{n} x_{i u} x_{j u} x_{i(n+u)} x_{j(n+u)}\right]\right) \\
+\left\{\sigma^{2}\left(1-\rho^{2}\right)\right\}^{-1}\left[\sum_{u=1}^{2 n} x_{i u}^{2} x_{j u}^{2}-\rho\left[\sum_{u=1}^{n} x_{i(n+u)}^{2} x_{j u}^{2}+\sum_{u=1}^{n} x_{i u}^{2} x_{j(n+u)}^{2}\right]\right]
\end{aligned}
$$

which implies to $c\left(\frac{2 \mathrm{n} \lambda_{4}}{\sigma^{2}\left(1-\rho^{2}\right)}\right)=\eta\left(2\left(\left(\frac{2 \mathrm{n} \lambda_{4}}{\sigma^{2}\left(1-\rho^{2}\right)}\right)+\left(\frac{2 \mathrm{n} \lambda_{4}}{\sigma^{2}\left(1-\rho^{2}\right)}\right)\right)\right.$

where $\mathrm{c}=3 \eta$ and $\mathrm{N}=2 \mathrm{n}$.

Where $\lambda_{2}$ and $\lambda_{4}$ constants and the summation are is over the design points.

The variances and covariances of the estimated parameters are,

$\mathrm{V}\left(\hat{\mathrm{b}}_{0}\right)=\frac{\lambda_{4}(\mathrm{c}+\mathrm{v}-1)(1+\rho) \sigma^{2}}{2 \mathrm{n} \Delta}$

$\mathrm{V}\left(\hat{\mathrm{b}}_{\mathrm{i}}\right)=\frac{\sigma^{2}\left(1-\rho^{2}\right)}{2 \mathrm{n} \lambda_{2}}$

$V\left(\hat{b}_{\mathrm{ij}}\right)=\frac{\sigma^{2}\left(1-\rho^{2}\right)}{2 \mathrm{n} \lambda_{4}}$

$\mathrm{V}\left(\hat{\mathrm{b}}_{\mathrm{ii}}\right)=\frac{\sigma^{2}\left(1-\rho^{2}\right)\left[\lambda_{4}(\mathrm{c}+\mathrm{v}-2)-(\mathrm{v}-1) \lambda_{2}^{2}(1-\rho)\right]}{(\mathrm{c}-1)(2 \mathrm{n}) \lambda_{4} \Delta}$

$\operatorname{Cov}\left(\hat{\mathrm{b}}_{0}, \hat{\mathrm{b}}_{\mathrm{ii}}\right)=\frac{-\lambda_{2} \sigma^{2}\left(1-\rho^{2}\right)}{2 \mathrm{n} \Delta}$

$\operatorname{Cov}\left(\hat{b}_{\mathrm{ii}}, \hat{b}_{\mathrm{jj}}\right)=\frac{\sigma^{2}\left(1-\rho^{2}\right)\left[\lambda_{2}^{2}(1-\rho)-\lambda_{4}\right]}{(\mathrm{c}-1)(2 \mathrm{n}) \lambda_{4} \Delta}$

Where $\Delta=\left[\lambda_{4}(\mathrm{c}+\mathrm{v}-1)-\mathrm{v} \lambda_{2}^{2}(1-\rho)\right]$ and other covariances are zero.

An inspection of the variance of $\widehat{b}_{0}$ shows that a necessary condition for the existence of a non-singular second order slope rotatable design under tri-diagonal structure is

$\left[\lambda_{4}(\mathrm{c}+\mathrm{v}-1)-\mathrm{v} \lambda_{2}^{2}(1-\rho)\right]>0$

$\frac{\lambda_{4}}{\lambda_{2}^{2}}>\frac{\mathrm{v}(1-\rho)}{(\mathrm{c}+\mathrm{v}-1)}$ (non - singularity condition)

For the second order model

$\frac{\partial \widehat{y}_{u}}{\partial x_{i}}=\hat{b}_{i}+2 \hat{b}_{i i} x_{i}+\sum_{j=1 ; j \neq i}^{v} \hat{b}_{i j} x_{j}$

$\mathrm{V}\left(\frac{\partial \widehat{\mathrm{y}}_{\mathrm{u}}}{\partial \mathrm{x}_{\mathrm{i}}}\right)=\mathrm{V}\left(\hat{\mathrm{b}}_{\mathrm{i}}\right)+4 \mathrm{x}_{\mathrm{i}}^{2} \mathrm{~V}\left(\hat{\mathrm{b}}_{\mathrm{ii}}\right)+\sum_{\mathrm{j}=1 ; \mathrm{j} \neq \mathrm{i}}^{\mathrm{v}} \mathrm{x}_{\mathrm{j}}^{2} \mathrm{~V}\left(\hat{\mathrm{b}}_{\mathrm{ij}}\right)$

The condition for right hand side of (25) to be a function of $\mathrm{d}^{2}=\sum_{\mathrm{i}=1}^{\mathrm{v}} \mathrm{x}_{\mathrm{i}}^{2}$ alone (for slope rotatability) is clearly,

$\mathrm{V}\left(\hat{\mathrm{b}}_{\mathrm{ii}}\right)=\frac{1}{4} \mathrm{~V}\left(\hat{\mathrm{b}}_{\mathrm{ij}}\right)$

(10) to (21) and (26) lead to condition

$\left(\frac{\mathrm{cN} \lambda_{4}}{\left(1-\rho^{2}\right)(1+\rho)}\right)\left[4 \mathrm{~N}-(\mathrm{c}+\mathrm{v}-2) \mathrm{N}+\mathrm{v}\left(\frac{\mathrm{N} \lambda_{2}^{2}(1-\rho)}{\lambda_{4}}\right)\right]$ 
$+\frac{\mathrm{N}^{2} \lambda_{4}}{\left(1-\rho^{2}\right)(1+\rho)}[5 \mathrm{v}-9]-\mathrm{N}^{2} \lambda_{2}^{2}\left[\frac{5 \mathrm{v}-4}{(1+\rho)^{2}}\right]=0$

Where $\mathrm{N}=2 \mathrm{n}$.

For $\rho=0$, equation (27) reduces to

$\lambda_{4}\left[\mathrm{v}(5-\mathrm{c})-(\mathrm{c}-3)^{2}\right]+\lambda_{2}^{2}[\mathrm{v}(\mathrm{c}-5)+4]=0$

This is similar to the SOSRD condition of uncorrelated errors case.

Therefore (10) to (21), (22) and (27) give a set of conditions for SOSRD under tri-diagonal correlated structure of errors for any general second order response surface design.

Further,

$\mathrm{V}\left(\frac{\partial \widehat{\mathrm{y}}_{\mathrm{u}}}{\partial \mathrm{x}_{\mathrm{i}}}\right)=\frac{\left(1-\rho^{2}\right)}{\mathrm{N}}\left(\frac{1}{\lambda_{2}}+\frac{\mathrm{d}^{2}}{\lambda_{4}}\right) \sigma^{2}$

\section{Construction of SOSRD under tri-diagonal correlated structure of errors using CCD}

Following Hader and Park [8], Das [4], [5], [7] methods of constructions, here a study on SOSRD under tri-diagonal correlated structure of errors using CCD is studied. The method of construction of SOSRD under tri-diagonal correlated structure of errors using CCD is given.

These designs are obtained by adding suitable factorial combinations to these obtained from $\frac{1}{2^{p}} \times 2^{v}$ fractional factorial design, where $\frac{1}{2^{p}} \times 2^{v}$ is a suitable fractional replicate of $2^{v}$, in which no interaction with less than five factors is confounded. In coded form CCD has the points of $2^{\mathrm{v}}\left(2^{\mathrm{t}(\mathrm{v})}\right)$ factorial with coordinates $( \pm 1, \pm 1, \ldots, \pm 1)$, and $2 \mathrm{v}$ axial points with coordinates

$\begin{array}{lllll} \pm \mathrm{a} & 0 & 0 & \ldots & 0 \\ 0 & \pm \mathrm{a} & 0 & \cdots & 0 \\ 0 & 0 & \pm \mathrm{a} & \ldots & 0 \\ \cdots & \cdots & \cdots & \cdots & \cdots \\ \cdots & \cdots & \cdots & \ldots & \ldots \\ 0 & 0 & 0 & \cdots & \pm \mathrm{a}\end{array}$

And at least one central point $(0,0,0, \cdots, 0)$ may be added if necessary. The central point may be replicated $\mathrm{n}_{0}$ times. Thus the total number of factorial combinations in this design is $2^{(\mathrm{v}-\mathrm{p})}+2 \mathrm{v}+\mathrm{n}_{0}$.

Here we consider a SRCCD of Hader and Park [8] having ' $n$ ' $\left(n=b 2^{t(k)}+2 v\right)$ non- central design points involving vfactors. The set of ' $n$ '-non central design points are extended to $2 n$ design points by adding ' $n$ ' $\left(n_{0}=n\right)$ central point's just below or above the ' $n$ ' non-central design points. Hence $2 n$ be the total number of design points of the SOSRD under tri-diagonal correlated structure of errors using CCD.

For the design points generated from the CCD, simple symmetry conditions (10) to (20) are true. Further, from (11), (12) and (13) we have

$\sum_{\mathrm{u}=1}^{2 \mathrm{n}} \mathrm{x}_{\mathrm{iu}}^{2}=2^{\mathrm{t}(\mathrm{v})}+2 \mathrm{a}^{2}=$ constant $=2 \mathrm{n} \lambda_{2}, 1 \leq \mathrm{i} \leq \mathrm{v}$

$\sum_{\mathrm{u}=1}^{2 \mathrm{n}} \mathrm{x}_{\mathrm{iu}}^{4}=2^{\mathrm{t}(\mathrm{v})}+2 \mathrm{a}^{4}=$ constant $=\mathrm{c}(2 \mathrm{n}) \lambda_{4}, 1 \leq \mathrm{i} \leq \mathrm{v}$

$\sum_{\mathrm{u}=1}^{2 \mathrm{n}} \mathrm{x}_{\mathrm{iu}}^{2} \mathrm{x}_{\mathrm{ju}}^{2}=2^{\mathrm{t}(\mathrm{v})}=$ constant $=2 \mathrm{n} \lambda_{4}, 1 \leq \mathrm{i} \neq \mathrm{j} \leq \mathrm{v}$

Substituting $\boldsymbol{\lambda}_{\mathbf{2}}, \boldsymbol{\lambda}_{\mathbf{4}}$ and $\mathrm{c}$ in equation (27), we get the following biquadratic equation,

$[8 \mathrm{v}(1-\rho)-4 \mathrm{~N}] \mathrm{a}^{8}+\left[2^{\mathrm{t}(\mathrm{v})+3} \mathrm{v}(1-\rho)\right] \mathrm{a}^{6}+$

$\left[2^{\mathrm{t}(\mathrm{v})+1}(4-\mathrm{v}) \mathrm{N}+2^{2 \mathrm{t}(\mathrm{v})+1} \mathrm{v}(1-\rho)+(1-\mathrm{v}) 2^{\mathrm{t}(\mathrm{v})+4}(1-\rho)\right] \mathrm{a}^{4}+\left[2^{2 \mathrm{t}(\mathrm{v})+4}(1-\mathrm{v})(1-\rho) \mathrm{a}^{2}\right]$

$+\left[2^{2 \mathrm{t}(\mathrm{v})+2}(\mathrm{v}-1) \mathrm{N}+2^{3 \mathrm{t}(\mathrm{v})+2}(1-\mathrm{v})(1-\rho)\right]=0$

The design exists, if at least one positive real root exits for equation (33). Solving equation (33) we get the SOSRD under tri-diagonal correlated structure of errors using CCD.

Example 1: We illustrate the above method with the construction of SOSRD under tri-diagonal correlated structure of errors using CCD for 3 - factors. Here $\mathrm{N}=2 \mathrm{n}=28$.

From equations (30), (31) and (32), we have

$\sum_{\mathrm{u}=1}^{2 \mathrm{n}} \mathrm{x}_{\mathrm{iu}}^{2}=8+2 \mathrm{a}^{2}=2 \mathrm{n} \lambda_{2}$

$\sum_{\mathrm{u}=1}^{2 \mathrm{n}} \mathrm{x}_{\mathrm{iu}}^{4}=8+2 \mathrm{a}^{4}=\mathrm{c}(2 \mathrm{n}) \lambda_{4}$

$\sum_{\mathrm{u}=1}^{2 \mathrm{n}} \mathrm{x}_{\mathrm{iu}}^{2} \mathrm{x}_{\mathrm{ju}}^{2}=8=2 \mathrm{n} \lambda_{4}$

From (35) and (36) we getc $=\frac{8+2 a^{4}}{8}$. Substituting for $\lambda_{2}, \lambda_{4}$ and $\mathrm{c}$ in (33) and on simplification, we get the following biquadratic equations for each value of $\rho$ in $a^{2}$ (viz.)

$[24(1-\rho)-112] \mathrm{a}^{8}+192(1-\rho) \mathrm{a}^{6}+[448+384(1-\rho)-256(1-\rho)] \mathrm{a}^{4}-$

$2048(1-\rho) a^{2}+[14336-4096(1-\rho)]=0$

(37) Has only one positive real root for each value of $\rho, a^{2}=4.0168$ (by taking $\rho=0.1$ ). It can be verified that (22) is also satisfied. This can be alternatively written directly from equation (27). Solving (27), we get a=2.0042 (by taking 
$\rho=0.1$ ), Substituting 'a' value in (34), (35) and (36) we obtain $\lambda_{2}=0.5726, \lambda_{4}=0.2857$ and c= 5.0337. From (21), we can obtain the variances and covariances. Further from (29), we have,

$\mathrm{V}\left(\frac{\partial \widehat{\mathrm{y}}_{\mathrm{u}}}{\partial \mathrm{x}_{\mathrm{i}}}\right)=\left(0.0617+0.1238 \mathrm{~d}^{2}\right) \sigma^{2}($ at $\rho=0.1)$.

\section{Example 2:}

We illustrate the above method with the construction of SOSRD under tri-diagonal correlated structure of errors using CCD for7- factors. Here $N=2 n=156$.

From equations (24), (25) and (26), we have

$\sum_{\mathrm{u}=1}^{2 \mathrm{n}} \mathrm{x}_{\mathrm{iu}}^{2}=64+2 \mathrm{a}^{2}=\mathrm{N}^{*} \lambda_{2}$

$\sum_{\mathrm{u}=1}^{2 \mathrm{n}} \mathrm{x}_{\mathrm{iu}}^{4}=64+2 \mathrm{a}^{4}=\mathrm{c} \mathrm{N}^{*} \lambda_{4}$

$\sum_{\mathrm{u}=1}^{2 \mathrm{n}} \mathrm{x}_{\mathrm{iu}}^{2} \mathrm{x}_{\mathrm{ju}}^{2}=64=\mathrm{N}^{*} \lambda_{4}$

From (39) and (40), we get $c=\frac{64+2 a^{4}}{64}$. Substituting for $\lambda_{2}, \lambda_{4}$ and $\mathrm{c}$ in (27) and on simplification, we get the following different biquadratic equations for each value of $\rho$ in $a^{2}$ (viz.)

$[56(1-\rho)-624] \mathrm{a}^{8}+[3584(1-\rho)] \mathrm{a}^{6}+[-59904+(57344(1-\rho)-6144(1-\rho))] \mathrm{a}^{4}-$

$393216(1-\rho) \mathrm{a}^{2}+[15335424-6291456(1-\rho)]=0$

(41) Has only one positive real root for each value of $\rho, a^{2}=11.0012$ (by taking $\rho=0.1$ ). It can be verified that (22) is also satisfied. This can be alternatively written directly from equation (27). Solving (27), we get $a=3.3168$ (by taking $\rho=0.1$ ) Substituting 'a' value in (38), (39) and (40) we obtain $\lambda_{2}=0.5513, \lambda_{4}=0.4103$ and $c=4.7820$. From (21), we can obtain the variances and covariances. Further from (29), we have,

$\mathrm{V}\left(\frac{\partial \widehat{\mathrm{y}}_{\mathrm{u}}}{\partial \mathrm{x}_{\mathrm{i}}}\right)=\left(0.0115+0.0155 \mathrm{~d}^{2}\right) \sigma^{2}($ at $\rho=0.1)$.

The variances of estimated slopes of these SOSRD under tri-diagonal correlated structure of errors using CCD for $-0.9 \leq \rho \leq 0.9$ and for " $v$ factors 2 to 8 "are given in appendix.

\section{Conclusions}

From Appendix table 1 and table 2 we observed that,

1) When the values of ' $\rho$ ' are increases slope rotatability value of " $a$ " is decreases for all the factors 2 to 8 .

2) We observed that the slope rotatability value of "a" at $\rho=-1$ which is equal to the SOSRD with errors are uncorrelated and homoscedastic estimated value at " 0 " central points.

At $\rho=0$ estimated value and slope rotatability derivative of SOSRD under tri-diagonal correlated structure is equal to the SOSRD uncorrelated errors case.

\section{References}

[1] Box, G.E.P. and Hunter, J.S. (1957). Multifactor experimental designs for exploring response surfaces. Annals of Mathematical Statistics, 28, 195-241.

[2] Das, R.N. (1997). Robust second order rotatable designs: Part-I (RSORD). Calcutta Statistical Association Bulletin, 47, 199-214.

[3] Das, R.N. (1999). Robust second order rotatable designs (Part -II). Calcutta Statistical Association Bulletin. 49, 65-78.

[4] Das, R. N. (2003a). Robust second order rotatable designs: Part -III. Journal of the Indian Society of Agricultural Statistics, 56, 117-130.

[5] Das, R.N. (2003b). Slope Rotatability with correlated errors: Calcutta Statistical Association Bulletin, 54, 57-70.

[6] Das, R. N. and Park, S. H. (2009). Measure of Robust slope rotatability for second order response surface designs, Journal of Applied Statistics, 36, 755-767.

[7] Das, R.N, Park, S.H., and Aggarwal, M. L. (2010). On D-optimal robust second order slope-rotatable designs, Journal of Statistical Planning and Inference, 140, 1269-1279.

[8] Hader, R.J. and Park, S.H. (1978). Slope rotatable central composite designs. Technometrics 20, 413-417.

[9] Panda, R.N. and Das, R.N. (1994). First order rotatable designs with correlated errors. Calcutta Statistical Association Bulletin, 44, 83-101.

[10] Park, S.H. (1987). A Class of multifactor designs for estimating the slope of response surfaces, Technometrics, 29, 449-453.

[11] Raghavarao, D. (1971). Constructions and combinatorial problems in design of experiments, John Wiley, New York. 


\section{Appendix}

Table 1: The Variance of Estimated Derivatives under Tri-Diagonal Correlated Structure of Errors Using CCD for the Factors $2 \leq \mathrm{v} \leq 8$

\begin{tabular}{|c|c|c|c|c|}
\hline \multirow{3}{*}{$\rho$} & \multicolumn{4}{|c|}{ Central composite designs } \\
\hline & \multicolumn{2}{|c|}{$\begin{array}{c}\mathrm{v}=2 \\
\mathrm{n}=8, \mathrm{~N}=2 \mathrm{n}=16\end{array}$} & \multicolumn{2}{|c|}{$\begin{array}{c}\mathrm{v}=3 \\
\mathrm{n}=14, \mathrm{~N}=2 \mathrm{n}=28\end{array}$} \\
\hline & $\hat{a}$ & $\mathrm{~V}\left(\frac{\partial \hat{\mathrm{y}}_{\mathrm{u}}}{\partial \mathrm{x}_{\mathrm{i}}}\right)$ & $\hat{a}$ & $\mathrm{~V}\left(\frac{\partial \hat{\mathrm{y}}_{\mathrm{u}}}{\partial \mathrm{x}_{\mathrm{i}}}\right)$ \\
\hline-1 & 2.2533 & - & 2.5551 & $0.0094 \sigma^{2}+0.0238 \sigma^{2} \mathrm{~d}^{2}$ \\
\hline-0.9 & 2.1756 & $0.0141 \sigma^{2}+0.0475 \sigma^{2} \mathrm{~d}^{2}$ & 2.4616 & $0.0187 \sigma^{2}+0.0450 \sigma^{2} \mathrm{~d}^{2}$ \\
\hline-0.8 & 2.1050 & $0.0280 \sigma^{2}+0.0900 \sigma^{2} \mathrm{~d}^{2}$ & 2.3772 & $0.0274 \sigma^{2}+0.0638 \sigma^{2} \mathrm{~d}^{2}$ \\
\hline-0.7 & 2.0411 & $0.0414 \sigma^{2}+0.1275 \sigma^{2} \mathrm{~d}^{2}$ & 2.3028 & $0.0355 \sigma^{2}+0.0800 \sigma^{2} \mathrm{~d}^{2}$ \\
\hline-0.6 & 1.9836 & $0.0539 \sigma^{2}+0.1600 \sigma^{2} \mathrm{~d}^{2}$ & 2.2386 & $0.0428 \sigma^{2}+0.0938 \sigma^{2} \mathrm{~d}^{2}$ \\
\hline-0.5 & 1.9321 & $0.0654 \sigma^{2}+0.1875 \sigma^{2} \mathrm{~d}^{2}$ & 2.1843 & $0.0490 \sigma^{2}+0.1050 \sigma^{2} \mathrm{~d}^{2}$ \\
\hline-0.4 & 1.8863 & $0.0756 \sigma^{2}+0.2100 \sigma^{2} \mathrm{~d}^{2}$ & 2.1390 & $0.0541 \sigma^{2}+0.1138 \sigma^{2} \mathrm{~d}^{2}$ \\
\hline-0.3 & 1.8457 & $0.0842 \sigma^{2}+0.2275 \sigma^{2} \mathrm{~d}^{2}$ & 2.1015 & $0.0579 \sigma^{2}+0.1200 \sigma^{2} \mathrm{~d}^{2}$ \\
\hline-0.2 & 1.8097 & $0.0910 \sigma^{2}+0.2400 \sigma^{2} \mathrm{~d}^{2}$ & 2.0703 & $0.0605 \sigma^{2}+0.1238 \sigma^{2} \mathrm{~d}^{2}$ \\
\hline-0.1 & 1.7781 & $0.0959 \sigma^{2}+0.2475 \sigma^{2} \mathrm{~d}^{2}$ & 2.0444 & $0.0618 \sigma^{2}+0.1250 \sigma^{2} \mathrm{~d}^{2}$ \\
\hline 0 & 1.7501 & $0.0988 \sigma^{2}+0.2500 \sigma^{2} \mathrm{~d}^{2}$ & 2.0226 & $0.0617 \sigma^{2}+0.1238 \sigma^{2} \mathrm{~d}^{2}$ \\
\hline 0.1 & 1.7254 & $0.0995 \sigma^{2}+0.2475 \sigma^{2} \mathrm{~d}^{2}$ & 2.0042 & $0.0603 \sigma^{2}+0.1200 \sigma^{2} \mathrm{~d}^{2}$ \\
\hline 0.2 & 1.7036 & $0.0979 \sigma^{2}+0.2400 \sigma^{2} \mathrm{~d}^{2}$ & 1.9884 & $0.0576 \sigma^{2}+0.1138 \sigma^{2} \mathrm{~d}^{2}$ \\
\hline 0.3 & 1.6843 & $0.0941 \sigma^{2}+0.2275 \sigma^{2} \mathrm{~d}^{2}$ & 1.9749 & $0.0535 \sigma^{2}+0.1050 \sigma^{2} \mathrm{~d}^{2}$ \\
\hline 0.4 & 1.6671 & $0.0879 \sigma^{2}+0.2100 \sigma^{2} \mathrm{~d}^{2}$ & 1.9632 & $0.0480 \sigma^{2}+0.0938 \sigma^{2} \mathrm{~d}^{2}$ \\
\hline 0.5 & 1.6517 & $0.0793 \sigma^{2}+0.1875 \sigma^{2} \mathrm{~d}^{2}$ & 1.9529 & $0.0411 \sigma^{2}+0.0800 \sigma^{2} \mathrm{~d}^{2}$ \\
\hline 0.6 & 1.6379 & $0.0683 \sigma^{2}+0.1600 \sigma^{2} \mathrm{~d}^{2}$ & 1.9438 & $0.0329 \sigma^{2}+0.0638 \sigma^{2} \mathrm{~d}^{2}$ \\
\hline 0.7 & 1.6255 & $0.0549 \sigma^{2}+0.1275 \sigma^{2} \mathrm{~d}^{2}$ & 1.9358 & $0.0233 \sigma^{2}+0.0450 \sigma^{2} \mathrm{~d}^{2}$ \\
\hline 0.8 & 1.6143 & $0.0391 \sigma^{2}+0.0900 \sigma^{2} \mathrm{~d}^{2}$ & 1.9287 & $0.0123 \sigma^{2}+0.0238 \sigma^{2} \mathrm{~d}^{2}$ \\
\hline 0.9 & 1.6042 & $0.0208 \sigma^{2}+0.0475 \sigma^{2} \mathrm{~d}^{2}$ & 1.9222 & \\
\hline
\end{tabular}

\begin{tabular}{|c|c|c|c|c|}
\hline \multirow{3}{*}{$\rho$} & \multicolumn{4}{|c|}{ Central composite designs } \\
\hline & \multicolumn{2}{|c|}{$\begin{array}{c}\mathrm{v}=4 \\
\mathrm{n}=24, \mathrm{~N}=2 \mathrm{n}=48\end{array}$} & \multicolumn{2}{|c|}{$\begin{array}{c}v=5 \\
n=26, N=2 n=52\end{array}$} \\
\hline & $\hat{a}$ & $\mathrm{~V}\left(\frac{\partial \hat{\mathrm{y}}_{\mathrm{u}}}{\partial \mathrm{x}_{\mathrm{i}}}\right)$ & $\hat{a}$ & $\mathrm{~V}\left(\frac{\partial \hat{\mathrm{y}}_{\mathrm{u}}}{\partial \mathrm{x}_{\mathrm{i}}}\right)$ \\
\hline-1 & 2.8798 & - & 2.9861 & - \\
\hline-0.9 & 2.7796 & $0.0060 \sigma^{2}+0.0119 \sigma^{2} \mathrm{~d}^{2}$ & 2.8344 & $0.0059 \sigma^{2}+0.0119 \sigma^{2} \mathrm{~d}^{2}$ \\
\hline-0.8 & 2.6911 & $0.0118 \sigma^{2}+0.0225 \sigma^{2} \mathrm{~d}^{2}$ & 2.7034 & $0.0118 \sigma^{2}+0.0225 \sigma^{2} d^{2}$ \\
\hline-0.7 & 2.6160 & $0.0172 \sigma^{2}+0.0319 \sigma^{2} \mathrm{~d}^{2}$ & 2.6016 & $0.0173 \sigma^{2}+0.0319 \sigma^{2} \mathrm{~d}^{2}$ \\
\hline-0.6 & 2.5547 & $0.0220 \sigma^{2}+0.0400 \sigma^{2} \mathrm{~d}^{2}$ & 2.5284 & $0.0222 \sigma^{2}+0.0400 \sigma^{2} \mathrm{~d}^{2}$ \\
\hline-0.5 & 2.5057 & $0.02638 \sigma^{2}+0.0469 \sigma^{2} d^{2}$ & 2.4770 & $0.0265 \sigma^{2}+0.0469 \sigma^{2} \mathrm{~d}^{2}$ \\
\hline-0.4 & 2.4668 & $0.0298 \sigma^{2}+0.0525 \sigma^{2} \mathrm{~d}^{2}$ & 2.4401 & $0.0301 \sigma^{2}+0.0525 \sigma^{2} \mathrm{~d}^{2}$ \\
\hline-0.3 & 2.4358 & $0.0327 \sigma^{2}+0.0569 \sigma^{2} \mathrm{~d}^{2}$ & 2.4130 & $0.0329 \sigma^{2}+0.0569 \sigma^{2} \mathrm{~d}^{2}$ \\
\hline-0.2 & 2.4109 & $0.0348 \sigma^{2}+0.0600 \sigma^{2} \mathrm{~d}^{2}$ & 2.3923 & $0.0350 \sigma^{2}+0.0600 \sigma^{2} \mathrm{~d}^{2}$ \\
\hline-0.1 & 2.3906 & $0.0361 \sigma^{2}+0.0619 \sigma^{2} \mathrm{~d}^{2}$ & 2.3762 & $0.0363 \sigma^{2}+0.0619 \sigma^{2} \mathrm{~d}^{2}$ \\
\hline 0 & 2.3738 & $0.0367 \sigma^{2}+0.0625 \sigma^{2} \mathrm{~d}^{2}$ & 2.3632 & $0.0368 \sigma^{2}+0.0625 \sigma^{2} \mathrm{~d}^{2}$ \\
\hline 0.1 & 2.3598 & $0.0365 \sigma^{2}+0.0619 \sigma^{2} \mathrm{~d}^{2}$ & 2.3527 & $0.0366 \sigma^{2}+0.0619 \sigma^{2} \mathrm{~d}^{2}$ \\
\hline 0.2 & 2.3479 & $0.0355 \sigma^{2}+0.0600 \sigma^{2} \mathrm{~d}^{2}$ & 2.3439 & $0.0356 \sigma^{2}+0.0600 \sigma^{2} \mathrm{~d}^{2}$ \\
\hline 0.3 & 2.3378 & $0.0338 \sigma^{2}+0.0569 \sigma^{2} \mathrm{~d}^{2}$ & 2.3364 & $0.0338 \sigma^{2}+0.0569 \sigma^{2} \mathrm{~d}^{2}$ \\
\hline 0.4 & 2.3290 & $0.0313 \sigma^{2}+0.0525 \sigma^{2} \mathrm{~d}^{2}$ & 2.3301 & $0.0313 \sigma^{2}+0.0525 \sigma^{2} \mathrm{~d}^{2}$ \\
\hline 0.5 & 2.3214 & $0.0280 \sigma^{2}+0.0469 \sigma^{2} \mathrm{~d}^{2}$ & 2.3246 & $0.0280 \sigma^{2}+0.0469 \sigma^{2} \mathrm{~d}^{2}$ \\
\hline 0.6 & 2.3147 & $0.0240 \sigma^{2}+0.0400 \sigma^{2} \mathrm{~d}^{2}$ & 2.3198 & $0.0239 \sigma^{2}+0.0400 \sigma^{2} \mathrm{~d}^{2}$ \\
\hline 0.7 & 2.3087 & $0.0191 \sigma^{2}+0.0319 \sigma^{2} \mathrm{~d}^{2}$ & 2.3155 & $0.0191 \sigma^{2}+0.0319 \sigma^{2} \mathrm{~d}^{2}$ \\
\hline 0.8 & 2.3034 & $0.0135 \sigma^{2}+0.0225 \sigma^{2} \mathrm{~d}^{2}$ & 2.3118 & $0.0135 \sigma^{2}+0.0225 \sigma^{2} \mathrm{~d}^{2}$ \\
\hline 0.9 & 2.2987 & $0.0072 \sigma^{2}+0.0119 \sigma^{2} \mathrm{~d}^{2}$ & 2.3084 & $0.0071 \sigma^{2}+0.0119 \sigma^{2} \mathrm{~d}^{2}$ \\
\hline
\end{tabular}




\begin{tabular}{|c|c|c|c|c|}
\hline \multirow{3}{*}{$\rho$} & \multicolumn{4}{|c|}{ Central composite designs } \\
\hline & \multicolumn{2}{|c|}{$\begin{array}{c}\mathrm{v}=6 \\
\mathrm{n}=44, \mathrm{~N}=2 \mathrm{n}=88\end{array}$} & \multicolumn{2}{|c|}{$\begin{array}{c}\mathrm{v}=7 \\
\mathrm{n}=78, \mathrm{~N}=2 \mathrm{n}=156\end{array}$} \\
\hline & â & $\mathrm{V}\left(\frac{\partial \hat{\mathrm{y}}_{\mathrm{u}}}{\partial \mathrm{x}_{\mathrm{j}}}\right)$ & â & $\mathrm{V}\left(\frac{\partial \hat{\mathrm{y}}_{\mathrm{u}}}{\partial \mathrm{x}_{\mathrm{i}}}\right)$ \\
\hline-1 & 3.3300 & - & 3.7510 & - \\
\hline-0.9 & 3.1896 & $0.0036 \sigma^{2}+0.0059 \sigma^{2} \mathrm{~d}^{2}$ & 3.6300 & $0.0021 \sigma^{2}+0.0030 \sigma^{2} \mathrm{~d}^{2}$ \\
\hline-0.8 & 3.0759 & $0.0071 \sigma^{2}+0.0113 \sigma^{2} d^{2}$ & 3.5399 & $0.0040 \sigma^{2}+0.0056 \sigma^{2} \mathrm{~d}^{2}$ \\
\hline-0.7 & 2.9927 & $0.0102 \sigma^{2}+0.0159 \sigma^{2} d^{2}$ & 3.4762 & $0.0058 \sigma^{2}+0.0080 \sigma^{2} \mathrm{~d}^{2}$ \\
\hline-0.6 & 2.9344 & $0.0130 \sigma^{2}+0.0200 \sigma^{2} \mathrm{~d}^{2}$ & 3.4317 & $0.0073 \sigma^{2}+0.0100 \sigma^{2} \mathrm{~d}^{2}$ \\
\hline-0.5 & 2.8935 & $0.0154 \sigma^{2}+0.0234 \sigma^{2} d^{2}$ & 3.3999 & $0.0086 \sigma^{2}+0.0117 \sigma^{2} \mathrm{~d}^{2}$ \\
\hline-0.4 & 2.8640 & $0.0174 \sigma^{2}+0.0263 \sigma^{2} d^{2}$ & 3.3765 & $0.0097 \sigma^{2}+0.0131 \sigma^{2} d^{2}$ \\
\hline-0.3 & 2.8420 & $0.0189 \sigma^{2}+0.0284 \sigma^{2} \mathrm{~d}^{2}$ & 3.3586 & $0.0105 \sigma^{2}+0.0142 \sigma^{2} \mathrm{~d}^{2}$ \\
\hline-0.2 & 2.8250 & $0.0200 \sigma^{2}+0.0300 \sigma^{2} d^{2}$ & 3.3447 & $0.0111 \sigma^{2}+0.0150 \sigma^{2} \mathrm{~d}^{2}$ \\
\hline-0.1 & 2.8116 & $0.0207 \sigma^{2}+0.0309 \sigma^{2} d^{2}$ & 3.3335 & $0.0115 \sigma^{2}+0.0155 \sigma^{2} \mathrm{~d}^{2}$ \\
\hline 0 & 2.8008 & $0.0210 \sigma^{2}+0.0313 \sigma^{2} \mathrm{~d}^{2}$ & 3.3244 & $0.0116 \sigma^{2}+0.0156 \sigma^{2} \mathrm{~d}^{2}$ \\
\hline 0.1 & 2.7919 & $0.0208 \sigma^{2}+0.0309 \sigma^{2} d^{2}$ & 3.3168 & $0.0115 \sigma^{2}+0.0155 \sigma^{2} \mathrm{~d}^{2}$ \\
\hline 0.2 & 2.7845 & $0.0202 \sigma^{2}+0.0300 \sigma^{2} d^{2}$ & 3.3104 & $0.0122 \sigma^{2}+0.0150 \sigma^{2} \mathrm{~d}^{2}$ \\
\hline 0.3 & 2.7782 & $0.0192 \sigma^{2}+0.0284 \sigma^{2} \mathrm{~d}^{2}$ & 3.3049 & $0.0106 \sigma^{2}+0.0142 \sigma^{2} \mathrm{~d}^{2}$ \\
\hline 0.4 & 2.7727 & $0.0177 \sigma^{2}+0.0263 \sigma^{2} d^{2}$ & 3.3002 & $0.0098 \sigma^{2}+0.0131 \sigma^{2} \mathrm{~d}^{2}$ \\
\hline 0.5 & 2.7680 & $0.0158 \sigma^{2}+0.0234 \sigma^{2} \mathrm{~d}^{2}$ & 3.2961 & $0.0087 \sigma^{2}+0.0117 \sigma^{2} d^{2}$ \\
\hline 0.6 & 2.7639 & $0.0135 \sigma^{2}+0.0200 \sigma^{2} d^{2}$ & 3.2924 & $0.0075 \sigma^{2}+0.0100 \sigma^{2} \mathrm{~d}^{2}$ \\
\hline 0.7 & 2.7603 & $0.0108 \sigma^{2}+0.0159 \sigma^{2} \mathrm{~d}^{2}$ & 3.2892 & $0.0060 \sigma^{2}+0.0080 \sigma^{2} \mathrm{~d}^{2}$ \\
\hline 0.8 & 2.7571 & $0.0076 \sigma^{2}+0.0113 \sigma^{2} d^{2}$ & 3.2864 & $0.0042 \sigma^{2}+0.0056 \sigma^{2} \mathrm{~d}^{2}$ \\
\hline 0.9 & 2.7542 & $0.0040 \sigma^{2}+0.0059 \sigma^{2} d^{2}$ & 3.2838 & $0.0022 \sigma^{2}+0.0030 \sigma^{2} \mathrm{~d}^{2}$ \\
\hline
\end{tabular}

\begin{tabular}{|c|c|c|}
\hline \multirow[t]{3}{*}{$\rho$} & \multicolumn{2}{|c|}{ Central composite designs } \\
\hline & \multicolumn{2}{|c|}{$\begin{array}{c}v=8 \\
n=80, \quad N=2 n=160\end{array}$} \\
\hline & $\hat{\mathbf{a}}$ & $v\left(\frac{\partial \hat{y}_{\mathbf{u}}}{\partial x_{\mathbf{i}}}\right)$ \\
\hline-1 & 3.8115 & $0.0021 \sigma^{2}+0.0030 \sigma^{2} \mathrm{~d}^{2}$ \\
\hline-0.9 & 3.6511 & $0.0040 \sigma^{2}+0.0056 \sigma^{2} \mathrm{~d}^{2}$ \\
\hline-0.8 & 3.5386 & $0.00583 \sigma^{2}+0.0080 \sigma^{2} \mathrm{~d}^{2}$ \\
\hline-0.7 & 3.4673 & $0.0073 \sigma^{2}+0.0100 \sigma^{2} \mathrm{~d}^{2}$ \\
\hline-0.6 & 3.4218 & $0.0086 \sigma^{2}+0.0117 \sigma^{2} d^{2}$ \\
\hline-0.5 & 3.3913 & $0.00971 \sigma^{2}+0.0131 \sigma^{2} \mathrm{~d}^{2}$ \\
\hline-0.4 & 3.3697 & $0.0105 \sigma^{2}+0.0142 \sigma^{2} \mathrm{~d}^{2}$ \\
\hline-0.3 & 3.3539 & $0.0111 \sigma^{2}+0.0150 \sigma^{2} d^{2}$ \\
\hline-0.2 & 3.3417 & $0.0115 \sigma^{2}+0.0155 \sigma^{2} \mathrm{~d}^{2}$ \\
\hline-0.1 & 3.3321 & $0.0116 \sigma^{2}+0.0156 \sigma^{2} \mathrm{~d}^{2}$ \\
\hline 0 & 3.3244 & $0.0115 \sigma^{2}+0.0155 \sigma^{2} d^{2}$ \\
\hline 0.1 & 3.3180 & $0.0112 \sigma^{2}+0.0150 \sigma^{2} \mathrm{~d}^{2}$ \\
\hline 0.2 & 3.3127 & $0.0106 \sigma^{2}+0.0142 \sigma^{2} \mathrm{~d}^{2}$ \\
\hline 0.3 & 3.3082 & $0.0098 \sigma^{2}+0.0131 \sigma^{2} d^{2}$ \\
\hline 0.4 & 3.3043 & $0.0087 \sigma^{2}+0.0117 \sigma^{2} d^{2}$ \\
\hline 0.5 & 3.3009 & $0.0075 \sigma^{2}+0.0100 \sigma^{2} \mathrm{~d}^{2}$ \\
\hline 0.6 & 3.2979 & $0.0059 \sigma^{2}+0.0080 \sigma^{2} \mathrm{~d}^{2}$ \\
\hline 0.7 & 3.2953 & $0.0042 \sigma^{2}+0.0056 \sigma^{2} \mathrm{~d}^{2}$ \\
\hline 0.8 & 3.2930 & $0.0022 \sigma^{2}+0.0030 \sigma^{2} \mathrm{~d}^{2}$ \\
\hline 0.9 & 3.2909 & $0.0021 \sigma^{2}+0.0030 \sigma^{2} \mathrm{~d}^{2}$ \\
\hline
\end{tabular}

